\title{
Correction: Water power to the people
}

Eric Smalley

Nature Energy 1, 16092 (2016); published 11 July 2016; corrected 25 July 2016.

In the original version of this Feature the proportion of global electricity production in 2013 that came from hydroelectric plants was misreported. The sentence should have read 'In 2013 hydroelectric plants generated 3,874 terawatt hours (TWh) of electricity worldwide, accounting for 16.3 per cent of global electricity production.' This error has been corrected in all versions of the Feature. 